\title{
Pulmonary vein isolation and autonomic denervation for the management of paroxysmal atrial fibrillation by a minimally invasive surgical approach
}

\author{
James R. Edgerton, MD, ${ }^{\mathrm{a}, \mathrm{b}}$ William T. Brinkman, MD, ${ }^{\mathrm{a}, \mathrm{c}}$ Tara Weaver, RN, ${ }^{\mathrm{a}}$ Syma L. Prince, RN, ${ }^{\mathrm{a}}$ \\ Daniel Culica, MD, ${ }^{a}$ Morley A. Herbert, PhD, ${ }^{\mathrm{c}}$ and Michael J. Mack, MD ${ }^{\mathrm{a}, \mathrm{c}}$
}

\begin{abstract}
Background: Advances in technology such as epicardial bipolar radiofrequency pulmonary vein isolation, ganglionated plexi identification, and isolation and thoracoscopic left atrial appendage exclusion have enabled less invasive surgical options for management of atrial fibrillation.
\end{abstract}

\begin{abstract}
Methods: We performed a prospective, nonrandomized study of consecutive patients with symptomatic paroxysmal atrial fibrillation undergoing a video-assisted, minimally invasive surgical ablation procedure. The procedure consisted of bilateral, epicardial pulmonary vein isolation with bipolar radiofrequency, partial autonomic denervation, and selective excision of the left atrial appendage. Minimum follow-up was 1 year with longterm monitoring (24-hour continuous, 14-day event or pacemaker interrogation).
\end{abstract}

\begin{abstract}
Results: Between March 2005 and January 2008, 52 patients (35 male), mean age 60.3 years (range, $42-79$ years) underwent the procedure. The left atrial appendage was isolated in $88.0 \%(44 / 50)$. Average hospital stay was 5.2 days (range 3-10 days). There were no operative deaths or major adverse cardiac events. On long-term monitoring, freedom from atrial fibrillation/flutter/tachycardia was $86.3 \%(44 / 51)$ and $80.8 \%(42 / 52)$ at 6 and 12 months, respectively. Antiarrhythmic drugs were stopped in 33 of 37 patients and warfarin in 30 of 37 of the patients in whom ablation was successful at 12 months. Freedom from symptoms attributed to atrial fibrillation/flutter/tachycardia was $78.0 \%(39 / 50)$ at 6 months and $63.8 \%(30 / 47)$ at 12 months.
\end{abstract}

Conclusions: Minimally invasive surgical ablation is effective in the management of paroxysmal atrial fibrillation as evidenced by freedom from atrial arrythmias by long-term monitoring at 12 months. Measuring success using clinical symptoms underestimated clinical success as compared with long-term monitoring. (J Thorac Cardiovasc Surg 2010;140:823-8)

\begin{abstract}
Atrial fibrillation (AF) is a superventricular tachyarrhythmia characterized by uncoordinated atrial activation and consequent deterioration of mechanical function. ${ }^{1}$ With an estimated prevalence of $0.4 \%$ to $1 \%$ in the general population, AF is the most common cardiac arrhythmia encountered in clinical practice. ${ }^{2} \mathrm{AF}$ is associated with an increased long-term risk of stroke, heart failure, and allcause mortality. ${ }^{3}$ Clinical symptoms associated with $\mathrm{AF}$ are quite variable in character and severity but are generally manifested as decreased energy level, dyspnea, and palpitations. Objectives for the management of AF are threefold: rate control, prevention of thromboembolism, and correction of the rhythm abnormality. So that these objectives can be achieved, most patients are managed with antiarrhythmic
\end{abstract}

\footnotetext{
From the Cardiopulmonary Research Science and Technology Institute (CRSTI), ${ }^{\mathrm{a}}$ Dallas, Tex, The Atrial Fibrillation Center at The Heart Hospital, ${ }^{\mathrm{b}}$ Plano, Tex, and the Medical City Dallas Hospital, ${ }^{\mathrm{c}}$ Dallas, Tex.

Disclosures: James R. Edgerton reports consulting and lecture fees from Atricure

Received for publication Feb 9, 2009; revisions received Nov 17, 2009; accepted for publication Nov 29, 2009; available ahead of print March 18, 2010.

Address for reprints: James R Edgerton, MD, 708 Alliance Blvd, Pavilion One, Suite

700, Plano, TX 75093 (E-mail: edgertonjr@aol.com).

$0022-5223 / \$ 36.00$

Copyright (C) 2010 by The American Association for Thoracic Surgery

doi:10.1016/j.jtcvs.2009.11.065
}

drugs (AADs) and anticoagulation to minimize the embolic and hemodynamic sequelae of the condition. ${ }^{1}$

Haissaguerre and associates ${ }^{4}$ initially described spontaneous initiation of AF by ectopic beats originating in the pulmonary veins. This led to the development of catheterbased treatment strategies with the goal of electrical isolation of the pulmonary veins. ${ }^{5}$ This technique was hampered by the technical difficulty of achieving transmural electrical isolation using endocardial catheter ablation. The feasibility of pulmonary vein isolation by a minimally invasive surgical approach was initially described by Wolf and associates. ${ }^{6}$ This procedure evolved into an epicardial bipolar radiofrequency ablation that includes isolation of the pulmonary vein antrum, detection and ablation of ganglionated plexi (GP), division of the ligament of Marshall, and removal of the left atrial appendage (LAA).

The importance of the autonomic nervous system in the initiation of AF was first described by Coumel ${ }^{7-9}$ in 1993. GPs, found in atrial fat pads, have subsequently been shown to play a role in initiation and maintenance of $\mathrm{AF} .{ }^{10,11}$ Moreover, the ablation of these GPs has been demonstrated to abolish vagally mediated $\mathrm{AF} .{ }^{12}$

In addition to the GP, the ligament of Marshall has been shown to contain sympathetic and parasympathetic nerve 


$$
\begin{aligned}
& \text { Abbreviations and Acronyms } \\
& \qquad \begin{aligned}
\text { AADs } & =\text { antiarrhythmic drugs } \\
\text { AF } & =\text { atrial fibrillation } \\
\text { GP } & =\text { ganglionated (ganglionic) plexi } \\
\text { LAA } & =\text { left atrial appendage } \\
\text { LTM } & =\text { long-term monitoring }
\end{aligned}
\end{aligned}
$$

trunks important to cardiac autonomic function. Moreover, patients with a well-developed ligament of Marshall have been found to have an increased incidence of arrythmogenic foci in the left superior pulmonary veins. ${ }^{13}$

Finally, to further enhance effectiveness in preventing thromboembolism, exclusion or removal of the LAA is optimal. This is critical because thrombus material associated with AF arises most commonly in the LAA. Decreased flow within the LAA during AF (as evidenced by the formation of spontaneous echo contrast or "smoke") has also been associated with embolic events. ${ }^{14}$

Herein, we describe our initial experience and outcomes with a minimally invasive surgical procedure of pulmonary vein isolation by bipolar radiofrequency, intraoperative confirmation of transmurality, mapping for and ablation of GPs, and exclusion of the LAA.

\section{METHODS}

A nonrandomized series of consecutive patients with symptomatic paroxysmal AF were entered into the study. The study was approved by the Institutional Review Boards of Medical City Hospital, Dallas, Texas, and Baylor University Hospital, Dallas, Texas, and informed consent was obtained from all patients. All definitions and reporting standards were in accordance with the 2007 Heart Rhythm Society (HRS)/European Heart Rhythm Association (EHRA)/European Cardiac Arrhythmia Society (ECAS) expert consensus statement for catheter and surgical ablation of $\mathrm{AF}^{15}$

\section{Surgical Procedure}

Surgery was performed with the patient under general anesthetia with double-lumen endotracheal intubation for selective pulmonary ventilation. Arterial blood pressure and pulmonary artery monitoring by a SwanGanz catheter (Edwards LifeSciences, Irvine, Calif) was performed in all patients. Transesophageal echocardiography was performed on the operating table before and during each procedure. In addition, patients with prior catheter ablations underwent computed tomography or magnetic resonance imaging of the left atrium before the procedure. The procedures were performed through bilateral $5-\mathrm{cm}$ anterior minithoracotomies in the third or fourth intercostal space. Video assistance with a 5-mm $30^{\circ}$ endoscope was used. A sensing device was used to detect atrial electrical activity conducted into the upper and lower right pulmonary veins and the bifurcation. High-frequency stimulation at 12 volts at a cycle length of $50 \mathrm{~ms}$ and a pulse width of 1 to $10 \mathrm{~ms}$ was used to map 13 areas for GPs (Figure 1). These locations were recorded for subsequent ablation. Detection and localization of GPs were determined by evidence of a vagal response as defined by an increase in the RR interval of greater than $50 \%$ from baseline. Circumferential dissection of the right pulmonary veins with a lighted dissector was then performed. A bipolar radiofrequency clamp (Atricure, Inc, Cincinnati, Ohio) was then guided around the veins. The bipolar radiofrequency clamp was then used to perform wide antrum circumferential ablation. The ablation energy was delivered 3 to 5 times. Entrance block was confirmed by sensing for atrial activity in the pulmonary veins. If atrial activity was detected, ablation was readministered until entrance block was confirmed. Any of the 13 GP areas that still showed evidence of vagal innervation after application of the ablation lines were then targeted for direct radiofrequency ablation using a monopolar pen. On completion, a small-caliber surgical drain was placed through the thoracoscopic site. The patient was then repositioned for optimal access to the left side. Mirror-image surgical incisions were made. Whereas the pericardium was opened anterior to the phrenic nerve on the right side, it was opened posterior to the phrenic nerve on the left side. The same sequence of pulmonary vein sensing for the detection of atrial activity was performed first, followed by high-frequency stimulation for detection of GPs, especially in the area of the ligament of Marshall. Areas that showed evidence of vagal autonomic innervation were recorded on a map that outlined 10 areas on the left side (Figure 2). The ligament of Marshall was then divided and the pulmonary veins encircled as on the right side. A bipolar radiofrequency clamp was then applied to perform wide antrum circumferential ablation. On confirmation of pulmonary vein entrance block and targeted autonomic denervation, a drain was placed on the left side.

After completion of the left-sided ablation, excision or exclusion of the LAA was performed selectively. A variety of techniques were used to exclude the appendage, including excision with an endoscopic stapler with or without bovine pericardial support or simply staple exclusion of the LAA. In cases in which it was deemed hazardous to perform the appendectomy owing to anatomic constraints, the appendage was left intact. For all patients having LAA exclusion, transesophageal echocardiography was used to confirm that the exclusion was successful. If $\mathrm{AF}$ was present at the end of the procedure, the patient was cardioverted to sinus rhythm. The general anesthetic was reversed and the patient was extubated in the operating room. The patient was monitored for the first 24 hours in the intensive care unit.

Postoperatively, patients were returned to the same antiarrhythmic drug regimen that they were using when seen preoperatively. This was restarted as soon as they were able to resume receiving oral medication and was continued for the first 30 to 90 days.

\section{Follow-up}

Continuous telemetric monitoring was performed throughout the hospital stay and a 12-lead electrocardiogram was obtained 1,3, 6, and 12 months after discharge. Long-term monitoring (LTM) by either a 24-hour Holter monitor, 2- to 3-week event monitoring, or interrogation of an implanted pacemaker was obtained at 6 and 12 months. The definition of success for the procedure was no episodes of AF/left atrial flutter/atrial tachycardia greater than 30 seconds. Patients were interviewed to determine resolution or persistence of symptoms related to AF. Patient data were recorded concurrently into a custom-computerized database. All patients were monitored by a dedicated AF nurse specialist and the operating surgeon and electrophysiologist. Preliminary 6-month data for a subset of these patients have been published previously. ${ }^{16}$

\section{RESULTS}

From March 2005 through January 2008, 52 patients with paroxysmal AF underwent the described ablation procedure. Patient demographics are listed in Table 1. All procedures were completed as planned with no mortality or major complications. The LAA was excised or stapled in $44(88.0 \%)$ of 50 patients. LAA exclusion was not performed if the operative surgeon deemed the LAA anatomy unsafe for endoscopic staple exclusion. There were no instances of significant postoperative bleeding, heart block, or phrenic 


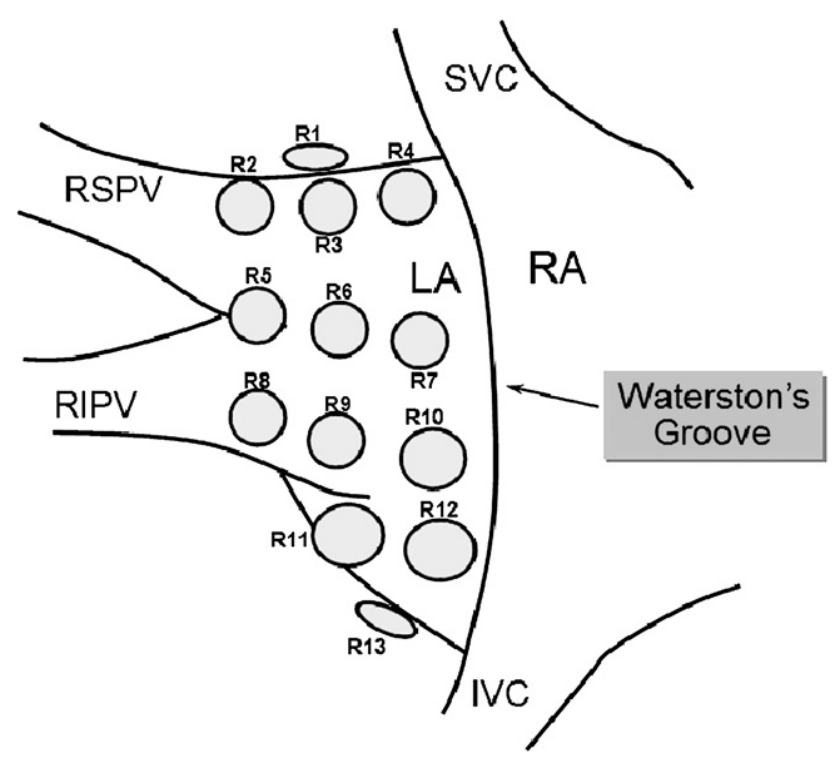

FIGURE 1. Ganglionated plexi on the right side. $R S P V$, Right superior pulmonary vein; $R I P V$, right interior pulmonary vein; $S V C$, superior vena cava; $I V C$, inferior vena cava; $R A$, right atrium; $L A$, left atrium; $R 1-R 13$, 13 areas for ganglionated plexi on the right side.

nerve injury. Average hospital stay was 5.2 days (range, 3-10 days). One case of temporary brachial plexus palsy related to intraoperative patient positioning was observed.

Three patients required new postoperative permanent pacemaker placement. The devices were placed for symp-

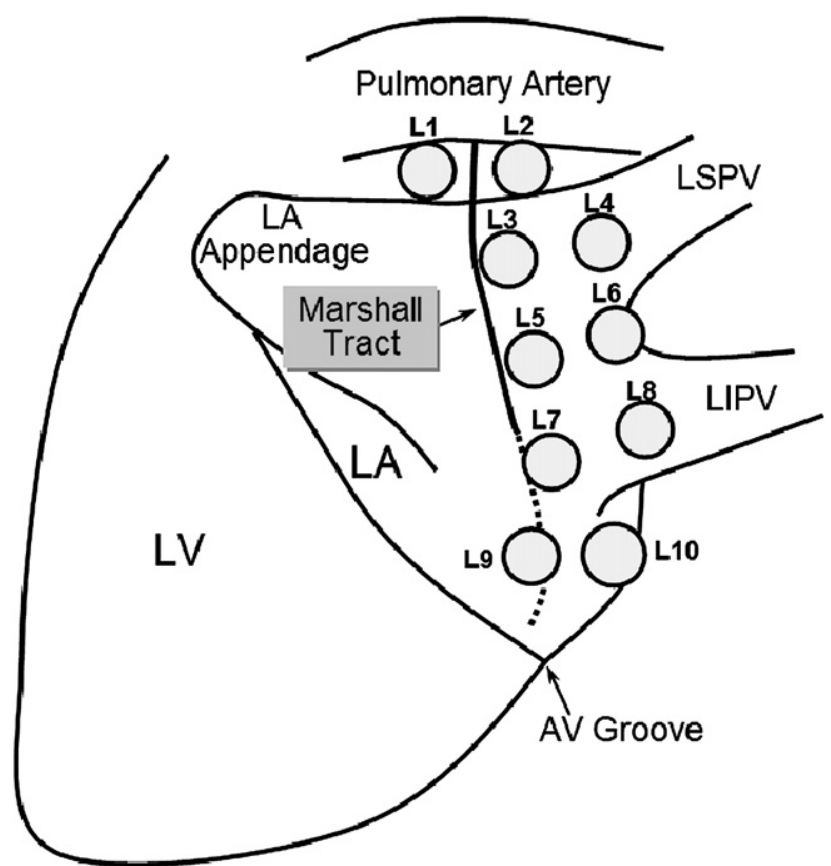

FIGURE 2. Ganglionated plexi on the left side. $L A$, Left atrium; $L V$, left ventricle; $L S P V$, left superior pulmonary vein; $L I P V$, left inferior pulmonary vein; $A V$, atrioventricular; $L 1-L 10 ; 10$ areas for ganglionated plexi on the left side.
TABLE 1. Patient demographics

\begin{tabular}{|c|c|}
\hline Characteristics & Results \\
\hline \multicolumn{2}{|l|}{ AF type: Paroxysmal } \\
\hline No. of patients & 52 \\
\hline Age (y) & 60.3 (range, 42-79) \\
\hline \multicolumn{2}{|l|}{ Gender } \\
\hline Male & $35(67.3 \%)$ \\
\hline Female & $17(32.7 \%)$ \\
\hline \multicolumn{2}{|l|}{ AF duration } \\
\hline$<6$ mo & $1(4.5 \%)$ \\
\hline $6-12 \mathrm{mo}$ & $0(0.0 \%)$ \\
\hline$>1 \mathrm{y}$ & $42(95.5 \%)$ \\
\hline Ejection fraction & 54.2 (range, $20-80$ ) \\
\hline Left atrial size & 4.8 (range, 3.7-6.0) \\
\hline \multicolumn{2}{|l|}{ Preoperative conditions } \\
\hline $\begin{array}{l}\text { Unsuccessful AAD therapy (amiodarone/ } \\
\text { sotalol/tikosin/rhythmol/flecanide) }\end{array}$ & $31(59.6 \%)$ \\
\hline Previous cardioversion & $19(36.5 \%)$ \\
\hline Warfarin intolerance/noncompliance & $4(9.8 \%)$ \\
\hline Previous catheter ablation & $10(20.8 \%)$ \\
\hline Preoperative pacemaker & $12(25.0 \%)$ \\
\hline Previous cardiac surgery & $9(18.8 \%)$ \\
\hline Coronary artery disease & $9(18.8 \%)$ \\
\hline \multicolumn{2}{|l|}{ Mitral regurgitation } \\
\hline None & $19(39.6 \%)$ \\
\hline Trace & $6(12.5 \%)$ \\
\hline Mild & $18(37.5 \%)$ \\
\hline Moderate & $5(10.4 \%)$ \\
\hline Severe & $0(0.0 \%)$ \\
\hline Previous transient ischemic attack & $2.1 \%$ \\
\hline \multicolumn{2}{|l|}{ Left atrial appendage isolation } \\
\hline Yes (amputation/stapling) & $44(88.0 \%)$ \\
\hline
\end{tabular}

tomatic bradycardia in the setting of a sick sinus syndrome. Two patients underwent additional electrophysiology ablation procedures after the operation for right atrial flutter. The patients were treated successfully and were free of AF/atrial flutter/atrial tachycardia by LTM at 12 months. Despite the success of the cardioversion, ablation was classed as a failure in these patients because flutter and fibrillation are both considered as failures of the procedure.

Long-term rhythm monitoring modalities (LTM) used at 6 months were 24-hour Holter monitor $(16 / 51 ; 31.4 \%)$, 14 day event recorder $(26 / 51 ; 51.0 \%)$, and pacemaker interrogation $(9 / 51 ; 17.7 \%)$.* $^{*}$

Long-term rhythm monitoring modalities (LTM) used at 12 months were 24-hour Holter monitor (5/52; 9.6\%), 14 day event recorder $(33 / 52 ; 63.5 \%)$, and pacemaker interrogation $(14 / 52 ; 26.9 \%)$.

Maintenance of normal sinus rhythm as demonstrated by LTM was $86.3 \%(44 / 51)$ at 6 months and $80.8 \%(42 / 52)$

\footnotetext{
* One patient at 6 months felt so good that she refused to wear a monitor. She did agree to the 12-month LTM follow-up.
} 
TABLE 2. Outcomes at 6 and 12 months by ECG and LTM

\begin{tabular}{lrr}
\hline & ECG & LTM \\
\hline 6 mo - NSR & $100 \%(50 / 50)$ & $86.3 \%(44 / 51)$ \\
NSR + off AAD & $77.6 \%(38 / 49)$ & $81.4 \%(35 / 43)$ \\
12 mo - NSR & $88.5 \%(46 / 52)$ & $80.8 \%(42 / 52)$ \\
NSR + off AAD & $87.8 \%(36 / 41)$ & $89.2 \%(33 / 37)$ \\
\hline$E C G$, Electrocardiogram; $L T M$, long-term monitoring; $N S R$, normal sinus rhythm; \\
$A A D$, antiarrhythmic drug.
\end{tabular}

at 12 months (Table 2). Of the patients in whom ablation was a success as defined by LTM, 81.4\% (35/43) were not receiving AADs at 6 months and 89.2\% (33/37) at 12 months. It should be noted that their preprocedure AAD dosing regimens were not altered after the procedure, unless they were stopped. In compliance with the HRS/EHRA/ECAS expert consensus statement AADs included the use of amiodarone, sotalol, or flecanide. ${ }^{15}$ Beta-blockers per say were not considered AADs. Of note, there was no attempt to discontinue AADs before the 6-month visit per follow-up protocol for this study. This lowered our clinical success rate off AAD at 6 months compared with 12 months (patients were generally taken off AAD at the 6-month visit, if appropriate).

Clinical symptoms attributed to AF were present in all patients before this procedure. Fifty patients at 6 months and 47 patients at 12 months completed the follow-up symptom surveys. Symptoms recorded as significant included fatigue, dyspnea, and palpitations. At 6 months and 12 months, 78.0\% (39/50) and 63.8\% (30/47) of patients were symptom-free, respectively. Interestingly, only 3 of the 11 symptomatic patients had clinical failure on LTM at 6 months, whereas 8 of the 17 symptomatic patients had clinical failure at 12 months. Of the 7 patients in AF on LTM at 6 months, $57.1 \%$ (4) reported they were without symptoms. The decision to discontinue the use of warfarin after clinical success was contingent on individual physician preference. At 12 months, 81.1\% (30/37) of the patients deemed to have had clinically successful ablation by LTM were not receiving warfarin.

We also compared LTM results at 6 and 12 months for each patient. Of 7 patients in AF at 6 months by LTM, 3 $(42.9 \%)$ were recorded as having normal sinus rhythm on LTM at 12 months. Conversely, 13.6\% (6/44) of the patients who were noted to be in normal sinus rhythm at 6 months on LTM were later noted to have reverted to AF at 12 months' follow-up with LTM.

\section{DISCUSSION}

This study demonstrates the feasibility and efficacy at 1 year of a minimally invasive surgical epicardial approach to the management of patients with paroxysmal AF. A number of aspects of this procedure are worthy of discussion. Core components of the procedure include bilateral pulmonary vein antral isolation with intraoperative documentation of block, detection of GPs with focal ablation, and excision of the LAA. It is our experience that documentation of conduction block is critical to the success of the procedure. Multiple applications of the energy source may be required to achieve entrance block. Because the veins may not be blocked with a single application, intraoperative testing for complete pulmonary vein isolation is critical for success. Whether acute block translates into chronic block cannot be determined within the confines of this study, but demonstration of acute entrance or exit block should be a mandatory component of the procedure. We used entrance block rather than exit block to be indicative of transmurality inasmuch as the mapping for GPs frequently stimulated the patients into AF. Use of entrance rather than exit block testing precludes the necessity for repeated intraoperative cardioversions.

The role of ablation of GPs in the management of AF continues to be debated. ${ }^{11,17}$ We performed mapping and ablation of GPs in all patients without randomization; therefore, it is not possible to know whether this contributed to the overall success rate of the procedure. Owing to the limitations of the current study, we cannot be certain that the targeted cell bodies were ablated. If only the axon was ablated and the nerve cell body remained viable, it is possible that subsequent reinervation may contribute to late failure. Indeed, we did have patients in whom ablation was a success at 6 months and a failure at 12 months. To help elucidate the role of the autonomic denervation with possible reinervation of the left atrium on success of ablation of AF, we have now initiated a program of performing full autonomic testing preoperatively and at 6 and 12 months postoperatively.

A component of this procedure was excision or exclusion of the LAA. Inasmuch as this was believed to be the most dangerous portion of the procedure to perform, we opted to leave the LAA intact in those patients in whom exclusion was believed to be high risk. Generally these were patients with friable tissues and a wide-based and short LAA. Although it is intuitive to surmise that exclusion of the LAA lowers the thromboembolic risk independent of the rhythm, that is by no means clear. ${ }^{18}$ Many patients have a strong desire to stop receiving anticoagulants. We stopped anticoagulant therapy in nearly $82 \%$ of our patients who were free of AF at 6 months and had no LAA. Discomfort with anticoagulant cessation on the part of the referring physician led to its continuance in a number of patients. Of note, there were no postoperative transient ischemic attacks or cerebrovascular accidents in this study. We are specifically interested in the role of warfarin cessation in patients with Cholesterol, Hypertension and Diabetes Study (CHADS) scores of 2 or greater after successful surgical AF ablation. ${ }^{1}$ This we hope will be answered in the setting of a multicenter trial of surgical ablation of paroxsysmal AF that is now ongoing.

This study also highlights the parameters to be used for defining "success.", We used the recent Heart Rhythm Society guidelines of no episodes of AF of greater than 
30 seconds by continuous or event monitoring. ${ }^{15}$ Clearly the use of isolated clinical follow-up or isolated interval electrocardiograms overestimates the success rate relative to rhythm detection by more intensive monitoring methods. In fact, had we determined success by interval electrocardiography, our success rate would have been $100 \%$ at 6 months' follow-up.

One could argue, however, that if the indication for the procedure is clinical symptoms and symptoms are relieved, the relevance of rhythm documentation is doubtful. Our data would argue against this assertion. It is remarkable that of the 11 patients with continued symptoms at 6 months, only 3 had clinical failure of ablation as defined by LTM. At 12 months only 8 patients of 17 with continued symptoms had clinical failure by LTM. Moreover, of the 7 patients with LTM failure at 6 months, only $3(42.9 \%)$ were symptomatic. This point highlights the lack of utility of clinical symptoms when making clinical management decisions for patients with paroxsysmal AF. Prior studies have shown that "palpitations" are often the result of atrial or ventricular premature beats and not recurrent $\mathrm{AF} .{ }^{19}$ Obtaining an accurate assessment of the efficacy of catheter and surgical ablation for the treatment of $\mathrm{AF}$ is quite difficult when reviewing the current medical literature. Reported series of single-procedure catheter-based pulmonary vein isolation used for the treatment of paroxsysmal AF vary widely in efficacy from $38 \%$ to $78 \%$. Methods of rhythm evaluation between studies also vary widely, ${ }^{15}$ as did the definition of "success" used to determine outcomes. Even with our study's adherence to the Heart Rhythm Society's reporting guidelines and use of LTM, our outcomes compare favorably with studies of catheter-based pulmonary vein isolation, even those using less stringent criteria for success and less intense monitoring.

\section{Limitations}

This study included only patients with paroxysmal AF, and the results cannot be extrapolated to persistent or long-standing persistent AF in which substrate remodeling occurs. $^{20,21}$ From our experience in these latter patients, the success rate is not as high and a more extensive set of ablation lines is necessary.

This study was not randomized to continuation of AADs; hence, the procedure's true efficacy cannot be determined with certainty. However, by our 12-month follow-up evaluation, $90 \%$ of patients deemed to have had a clinically successful ablation by LTM were receiving no AADs. This fact would argue against a major confounding bias of AADs. It is therefore likely that a treatment benefit resulted. It is also not known whether success at 1 year translates to longer term freedom from AF. In accordance with the Heart Rhythm Society's reporting guidelines, we plan annual follow-up with monitoring to determine this. This study also comprised a relatively small number of patients; whether these results are reproducible in larger cohorts of patients is unknown. The relative benefit of this procedure compared with catheter ablation is also unknown. Inasmuch as success rates of serial catheter-based ablations are reported to approach these results, a randomized trial of the 2 approaches is probably now appropriate.

In this study we chose to include patients who had a previous cardioversion, because when examined for the study, they exhibited typical paroxysmal pattern AF. The consensus statement includes the statement, "As noted in the ACC/AHA/ESC 2006 Guidelines, it is recognized that a particular patient may have AF episodes that fall into one or more of these categories. It is recommended that patients be categorized by their most frequent pattern of AF." This describes these patients-repeated intermittent episodes of more than 30 seconds characteristic of paroxysmal AF. Also, by including the patients in whom normal sinus rhythm had failed to develop despite an earlier cardioversion attempt, we are using a patient group that represents a greater challenge for the surgical ablation procedure and may reduce our success rate.

\section{CONCLUSIONS}

Minimally invasive surgical ablation is effective in the management of paroxysmal $\mathrm{AF}$ as evidenced by freedom from atrial arrythmias by scheduled LTM at 6 months and 1 year. The use of electrocardiographic analysis alone overestimated success by up to $15 \%$. The use of clinical symptoms underestimated clinical success as compared with LTM.

\section{References}

1. Fuster V, Rydén LE, Cannom DS, Crijns HJ, Curtis AB, Ellenbogen KA, et al. ACC/AHA/ESC 2006 Guidlines for the management of patients with atrial fibrillation. Circulation. 2006;114:e257-354.

2. Go A, Hylek E, Phillips K, Chang Y, Henault LE, Selby JV, et al. Prevalence of diagnosed atrial fibrillation in adults: national implications for rhythm management and stroke prevention: the AnTicoagulation and Risk Factors in Atrial Fibrillation (ATRIA) Study. JAMA. 2001;285:2370-5.

3. Stewart S, Hart CL, Hole DJ, McMurray JJ. A population-based study of the long term risks associated with atrial fibrillation: 20-year follow-up of the Renfrew/ Paisley study. Am J Med. 2002;113:359-64.

4. Haissaguerre M, Jais P, Shah D, Takahashi A, Hocini M, Quiniou G, et al. Spontaneous initiation of atrial fibrillation by ectopic beats originating in the pulmonary veins. $N$ Engl J Med. 1998;339:659-66.

5. Pappone C, Rosanio S, Oreto G, Tocchi M, Gugliotta F, Vicedomini G, et al. Circumferential radiofrequency ablation of pulmonary vein ostia: a new anatomic approach for curing atrial fibrillation. Circulation. 2000;102:2619-28.

6. Wolf R, Schneeberger E, Osterday R, Miller D, Merrill W, Flege JB Jr, et al. Video-assisted bilateral pulmonary vein isolation and left atrial appendage exclusion for atrial fibrillation. J Thorac Cardiovasc Surg. 2005;130:797-802.

7. Coumel P. Cardiac arrhythmias and the autonomic nervous system. J Cardiovasc Electrophysiol. 1993;4:338-55.

8. Coumel P. Paroxsysmal atrial fibrilation: a disorder of autonomic tone. Eur Heart J. 1994;15(Suppl A):9-16.

9. Coumel P. Autonomic influence in atrial tachyarrhythmias. J Cardiovasc Electrophysiol. 1996;7:999-1004.

10. Schauerte P, Scherlag BJ, Patterson E, Scherlag MA, Matsudaria K, Nakagawa $\mathrm{H}$, et al. Focal atrial fibrillation: experimental evidence for a pathophysiological role of the autonomic nervous system. J Cardiovasc Electrophysiol. 2001;12:592-9. 
11. Scherlag BJ, Po S. The intrinsic cardiac nervous system and atrial fibrillation. Curr Opin Cardiol. 2006;21:51-4.

12. Schauerte P, Scherlag B, Pitha J, Scherlag MA, Reynolds D, Lazzara R, et al. Catheter ablation of cardiac autonomic nerves for prevention of vagal atrial fibrillation. Circulation. 2000;102:2744-80.

13. Kurotabi T, Ito H, Inoue K, Iwakura K, Kawano S, Okamura A, et al. Marshall vein as arrhythmogenic source in patients with atrial fibrillation: correlation between its anatomy and electrophysiologic findings. J Cardiovasc Electrophysiol. 2006; 17:1062-8.

14. Fatkin D, Kelly RP, Feneley MP. Relations between left atrial appendage blood flow velocity, spontaneous echocardiographic contrast and thromboembolic risk in vivo. J Am Coll Cardiol. 1994;23:961-9.

15. Calkins H, Brugada J, Packer D, Cappato R, Chen S, Crijns H, et al. HRS/EHRA/ ECAS expert consensus statement on catheter and surgical ablation of atrial fibrillation: Recomendations for personnel, policy, procedures and follow-up. Heart Rhythm. 2007;4:816-61.
16. Edgerton JR, Edgerton ZJ, Weaver T, Reed K, Prince S, Herbert MA, et al. Minimally invasive pulmonary vein isolation and partial autonomic denervation for surgical treatment of atrial fibrillation. Ann Thorac Surg. 2008;86:35-9.

17. Scherlag B, Nakagawa H, Jackman W, Yamanashi WS, Patterson E, Po S, et al. Electrical stimulation to identify neural elements on the heart: Their role in atrial fibrillation. J Interv Card Electrophysiol. 2005;13:37-42.

18. Halperin J, Hart R. Atrial fibrillation and stroke: new ideas, persisting dilemmas. Stroke. 1988;19:937-41.

19. Vasamreddy CR, Dalal D, Dong J, Cheng A, Spragg D, Lamiy SZ, et al. Symptomatic and asymptomatic atrial fibrillation in patients undergoing radiofrequency catheter ablation. J Cardiovasc Electrophysiol. 2006;17:134-9.

20. Ausma J, Wijffels M, Thoné F, Wouters L, Allessie M, Borgers M. Structural changes of atrial myocardium due to sustained atrial fibrillation in the goat. Circulation. 1997;96:3157-63.

21. Brundel BJ, Henning RH, Kampinga HH, Van Gelder IC, Crijns HJ. Molecular mechanisms of remodeling in human atrial fibrillation. Cardiovasc Res. 2002;54:315-24. 